\title{
Large field-induced strains in a lead-free piezoelectric material
}

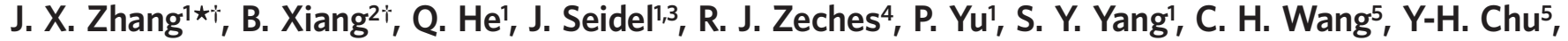 \\ L. W. Martin', A. M. Minor ${ }^{2,4}$ and R. Ramesh ${ }^{1,3,4}$
}

Piezoelectric materials exhibit a mechanical response to electrical inputs, as well as an electrical response to mechanical inputs, which makes them useful in sensors and actuators'. Lead-based piezoelectrics demonstrate a large mechanical response, but they also pose a health risk $^{2}$. The ferroelectric $\mathrm{BiFeO}_{3}$ is an attractive alternative because it is lead-free, and because strain can stabilize $\mathrm{BiFeO}_{3}$ phases with a structure that resembles a morphotropic phase boundary ${ }^{3}$. Here we report a reversible electric-field-induced strain of over $5 \%$ in $\mathrm{BiFeO}_{3}$ films, together with a characterization of the origins of this effect. In situ transmission electron microscopy coupled with nanoscale electrical and mechanical probing shows that large strains result from moving the boundaries between tetragonal- and rhombohedral-like phases, which changes the phase stability of the mixture. These results demonstrate the potential of $\mathrm{BiFeO}_{3}$ as a substitute for leadbased materials in future piezoelectric applications.

Over the past century, researchers have made great efforts to engineer and create materials with a significant mechanical response for applications ranging from sensing to actuation, and including the study of magnetostrictive compounds ${ }^{4}$, shape memory alloys (SMAs) ${ }^{5,6}$ and piezoelectrics ${ }^{1}$. Among these, piezoelectrics have become widely used due to their superior high-frequency response and force generation ${ }^{7}$. Piezoelectric materials with compositions near the morphotropic phase boundary (MPB) usually exhibit an anomalously high electromechanical coupling response. This discovery triggered a race for large piezoelectric constants and electric-fieldinduced strains (the key factors for actuation) in single crystals of lead-based relaxor perovskites such as $(1-x)\left[\mathrm{Pb}\left(\mathrm{Mg}_{1 / 3} \mathrm{Nb}_{2 / 3}\right) \mathrm{O}_{3}\right]-$ $x\left[\mathrm{PbTiO}_{3}\right], \quad(1-x)\left[\mathrm{Pb}\left(\mathrm{Zn}_{1 / 3} \mathrm{Nb}_{2 / 3}\right) \mathrm{O}_{3}\right]-x\left[\mathrm{PbTiO}_{3}\right]^{8,9}$, and MPBbased ceramics such as $\mathrm{Pb}\left(\mathrm{Zr}_{x} \mathrm{Ti}_{1-x}\right) \mathrm{O}_{3}$ (ref. 10). More recently, Ahart and colleagues ${ }^{11}$ observed a pressure-induced MPB in pure $\mathrm{PbTiO}_{3}$, suggesting an alternative to compositionally driven MPBs in complex relaxor systems. Their experimental observation and theoretical prediction ${ }^{12}$ opened a new field of research into high-performance electromechanical materials. Because of the toxicity of lead, it was also of great interest to investigate new possibilities for the presence of MPBs in simple, lead-free systems ${ }^{2,13}$. It has been reported that epitaxial strain can be used to stabilize different polymorphs of the ferroelectric multiferroic $\mathrm{BiFeO}_{3}$ (BFO), including a similar boundary between a tetragonal-like (T-) and rhombohedral-like (R-) phases ${ }^{3,14,15}$. We show that a large electromechanical response can be obtained by electrically controllable interconversion of two phases at the nanoscale. A reversible electric-field-induced strain of over
$5 \%$ is achieved, which is larger than values seen in applications using traditional piezoelectrics $\left(0.2 \%\right.$ in $\mathrm{Pb}\left(\mathrm{Zr}_{x} \mathrm{Ti}_{1-x}\right) \mathrm{O}_{3}, \quad 1.7 \%$ in ferroelectric relaxors, and $0.06 \%$ in lead-free ceramics ${ }^{1,11}$ ), making its strain value comparable to those of wellknown SMAs ${ }^{16,17}$.

The path to achieving large strains began with an investigation of the electromechanical coupling behaviour of macroscopic capacitors in a number of BFO samples, including those of pure R-phase and pure T-phase, as well as those with a mixture of both phases (for growth conditions see Methods). The effective converse piezoelectric coefficient $\left(d_{33}\right)$ in a macroscopic capacitor strongly depends on the structure of its films, as has been demonstrated in earlier studies of lead-based piezoelectrics ${ }^{1}$. As shown in Fig. 1a, a $d_{33}$ value of $\sim 53 \mathrm{pm} \mathrm{V}^{-1}$ was observed from samples with pure $\mathrm{R}$-phase and is consistent with work reported previously ${ }^{18,19}\left(d_{33}\right.$ measurement details are given in the Methods). In contrast, the $d_{33}$ value for BFO films of pure T-phase was found to be $\sim 30 \mathrm{pm} \mathrm{V}^{-1}$, determined by the relationship between its crystal orientation and the polar axis ${ }^{20}$. However, a strong increase of $d_{33}$ to a value of $\sim 115 \mathrm{pm} \mathrm{V}^{-1}$ was obtained in mixed-phase films. This suggests that, similar to findings for lead-based thin film systems near their classic, compositionally driven $\mathrm{MPB}^{21}$, a large electromechanical coupling effect can be obtained in BFO films having an MPB-like feature consisting of a nanoscale phase mixture. Further enhancement of $d_{33}$ in such samples is obtained through the removal of substrate clamping effects by reducing the lateral size of the capacitor ${ }^{22-24}$. Atomic force microscopy (AFM) studies (Fig. 1b), in conjunction with d.c. field switching, provides a first glimpse into the possible origins of the enhanced piezoelectric responses. The image in Fig. 1b shows the reversible conversion of stripe-like features (in the red box) corresponding to intimately mixed $\mathrm{T}$ - and R-phases and the flat features (in the blue box) corresponding to purely T-phase regions. The microscopic origin of this effect is the central focus of this paper.

The local electromechanical response in the mixed-phase BFO films was characterized by measuring piezoresponse hysteresis loops using a conductive AFM tip (Fig. 2a, inset). Electric fields (a.c. and d.c.) were simultaneously applied to acquire the piezoresponse hysteresis loops. The same experiment was carried out on a $\mathrm{Pb}\left(\mathrm{Zr}_{0.2} \mathrm{Ti}_{0.8}\right) \mathrm{O}_{3}(\mathrm{PZT})$ film under identical conditions (as a reference system), giving a $d_{33}$ value of $65 \mathrm{pm} \mathrm{V}^{-1}$. The maximum piezoresponse amplitude of the mixed-phase BFO films is almost four times higher than that from the PZT film. The relationship between the $d_{33}$, dielectric constant, tip-sample interaction and

'Department of Physics, University of California, Berkeley, California 94720, USA, ${ }^{2}$ National Center for Electron Microscopy, Lawrence Berkeley National Laboratory, Berkeley, California 94720, USA, ${ }^{3}$ Materials Science Division, Lawrence Berkeley National Laboratory, Berkeley, California 94720, USA, ${ }^{4}$ Department of Materials Science and Engineering, University of California, Berkeley, California 94720, USA, ${ }^{5}$ Department of Materials Science and Engineering, National Chiao Tung University, HsinChu, Taiwan 30010, ${ }^{6}$ Department of Materials Science and Engineering, University of Illinois, Urbana-Champaign, Urbana, Illinois 61801, USA; ‘These authors contributed equally to this work. *e-mail: jinxing@berkeley.edu 

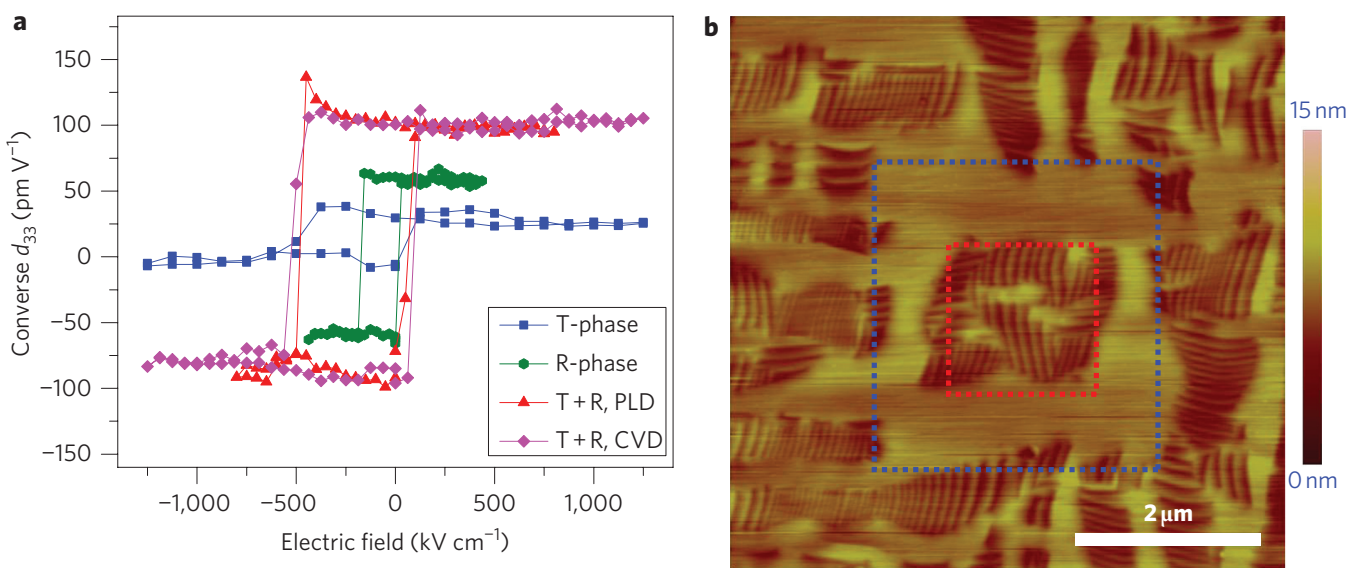

Figure 1 | Piezoelectric response in BFO capacitors. a, Piezoelectric hysteresis loops from T-like (blue curve), R-like (green curve) and mixed-phase (purple and red curves) BFO thin films, grown by pulsed laser deposition (PLD) and chemical vapor deposition (CVD), measured in capacitors with a diameter of $32 \mu \mathrm{m}$. b. High-resolution AFM images of an electric-field-induced phase transition between mixed phase and T-phase. The blue outlined area indicates a pure T-phase converted from mixed phase when subjected to a scanning probe electric field of $+1,500 \mathrm{kV} \mathrm{cm}^{-1}$. The red outlined area indicates a recovery of the mixed phase from pure T-phase with a probe electric field of $-600 \mathrm{kV} \mathrm{cm}^{-1}$.
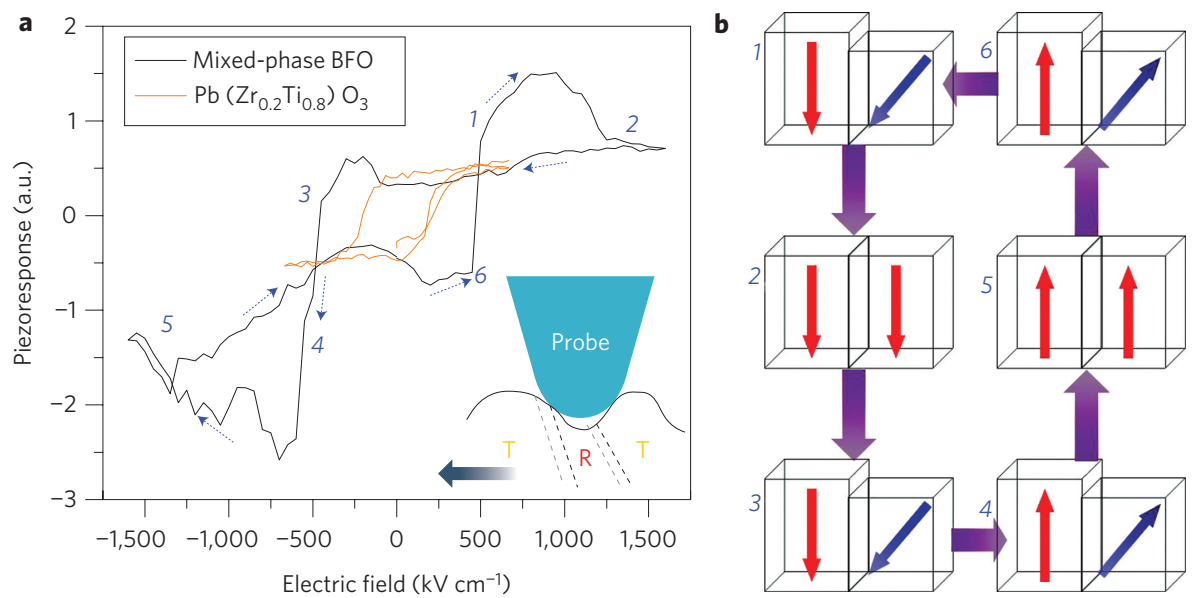

Figure 2 | Nanoscale localized switching. a, Piezoresponse hysteresis loops of a mixed-phase BFO film and a $\mathrm{Pb}\left(\mathrm{Zr}_{0.2} \mathrm{Ti}_{0.8}\right) \mathrm{O}_{3}$ film (the inset shows the interaction of the piezoresponse force microscopy cantilever and the mixed-phase BFO thin films). $\mathbf{b}$, Schematic of the polarization reversal and phase transition of the BFO film with a mixture of two phases under an electric field. The red arrow indicates the polarization of T-like phase and the blue arrow indicates the polarization of R-like phase.

piezoresponse amplitude of the films is described by ${ }^{25}$

$$
d_{33}=\frac{\Delta Z \int_{0}^{r_{0}} r \mathrm{~d} r}{\varepsilon_{r} \int_{0}^{r_{0}} \int_{0}^{t} E r \mathrm{~d} t \mathrm{~d} r}
$$

where $d_{33}$ is the effective converse piezoelectric coefficient, $\Delta Z$ is proportional to the piezoresponse amplitude, $\varepsilon_{\mathrm{r}}$ is the dielectric constant of the material, and $t$ is the film thickness. If we assume the geometry and the superposition of the probe and surface of the film to be approximately constant, $d_{33}$ will be predominantly determined by the values of $\varepsilon_{\mathrm{r}}(\sim 200 \text { for PZT, } \sim 100 \text { for BFO })^{26,27}$ and $\Delta Z$ under the electric field. We can therefore estimate that $d_{33}$ due to the phase transition is $\sim 520 \mathrm{pm} \mathrm{V}^{-1}$ for the mixed-phase BFO films.

We now focus on the microscopic details of the local piezoresponse hysteresis loops of the mixed-phase BFO samples, which show several distinct changes as a function of electric field. These features were qualitatively repeatable over several randomly chosen points on the sample surface (Supplementary Fig. S1). Careful examination of the loop in Fig. 2a allows the identification of six possible segments. Application of an electric field in the positive direction to the as-grown mixed $T+R$ state results in a large displacement, which we have interpreted as a consequence of the progressive conversion of this nanoscale mixed phase into a pure T-phase ${ }^{28}$. Once this phase mixture is fully converted (point 2 on the loop), the piezoresponse decreases. On reversal of the electric field, the piezoresponse remains at this low value until points 3 and 4 , when the mixed phase is formed again (with the polarization direction opposite to the as-grown state at point 4). Points 5 and 6 trace the subsequent evolution of the piezoresponse when the electric field is further increased in the negative direction and then reverses to the positive side. The possible atomic-scale changes corresponding to the sequence of events are schematically described by the six structural models in Fig. 2b. In the hysteresis curve of the PZT film in Fig. 2a, no dramatic increase of the piezoresponse amplitude like that in the curve of the mixed-phase BFO film can be seen.

We now investigate displacement at a fixed spatial location during a transition from the mixed state to the pure T-phase. Figure $3 a-c$ presents a sequence of AFM images of the same location as it is electrically switched from a mixed phase to a pure T-phase. This reversible movement of the phase boundary is accompanied by a 5-6 nm surface displacement for a film with a thickness of 


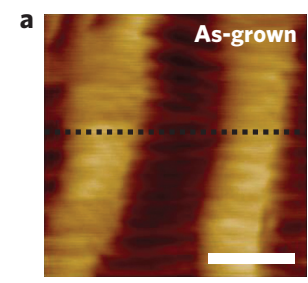

b
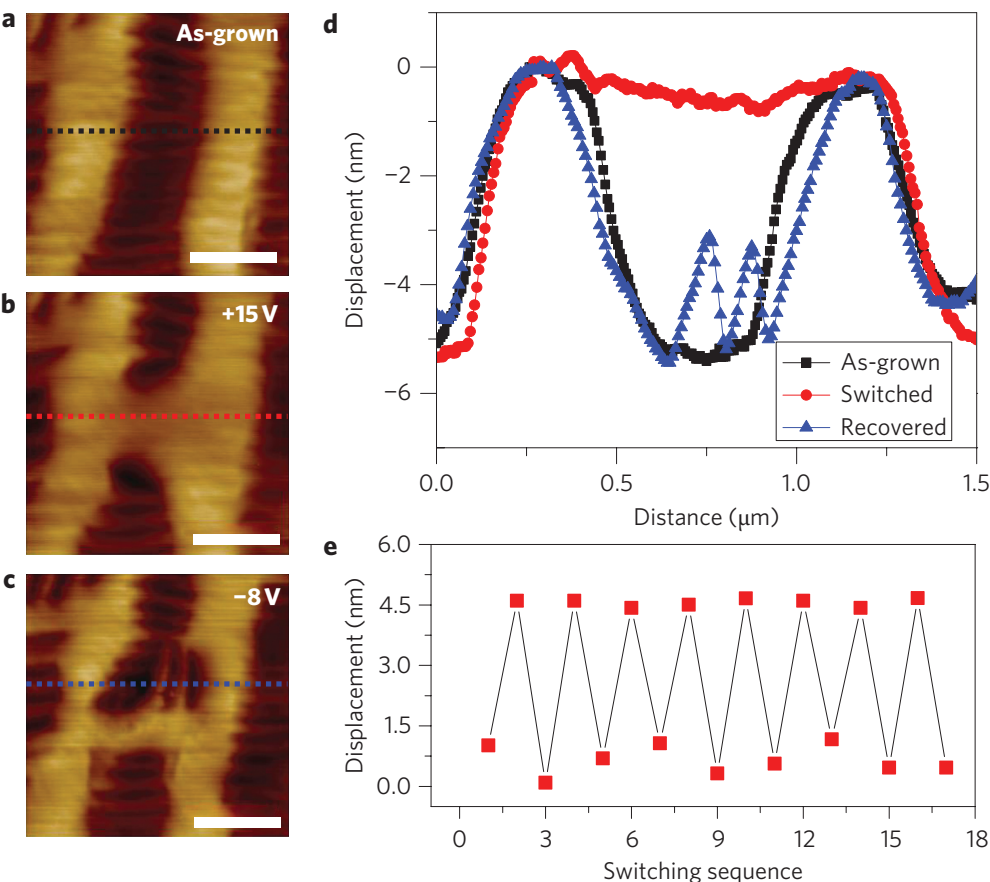

Figure 3 | Nanoscale reversible large electric-field-induced strain. a-c, Sequence of AFM images of the same location as it is electrically switched from a mixed phase to a pure T-phase: $\mathbf{a}$, topography of the as-grown film across the phase boundary; $\mathbf{b}$, switched by a positive electric field in an area of $500 \times 500 \mathrm{~nm}^{2}$; c, poled by a negative electric field in the same area. Scale bars, $500 \mathrm{~nm}$. d, Corresponding line scan profiles across regions of the as-grown (black line), switched (red line) and recovered (blue line) film. e, Repeatability of the large surface displacement.

a

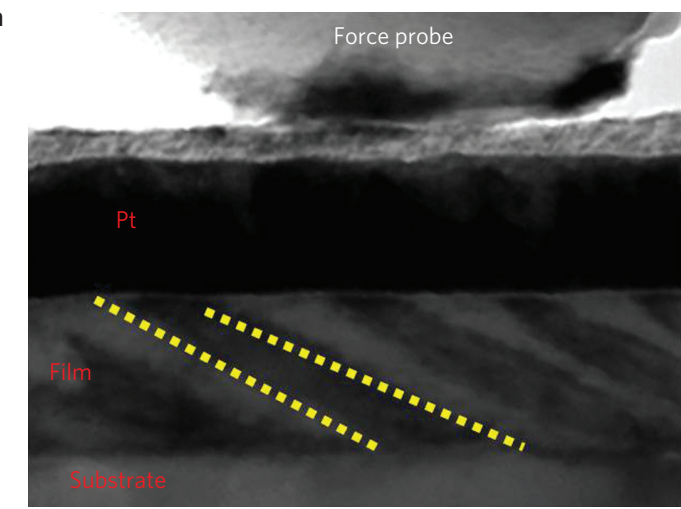

b

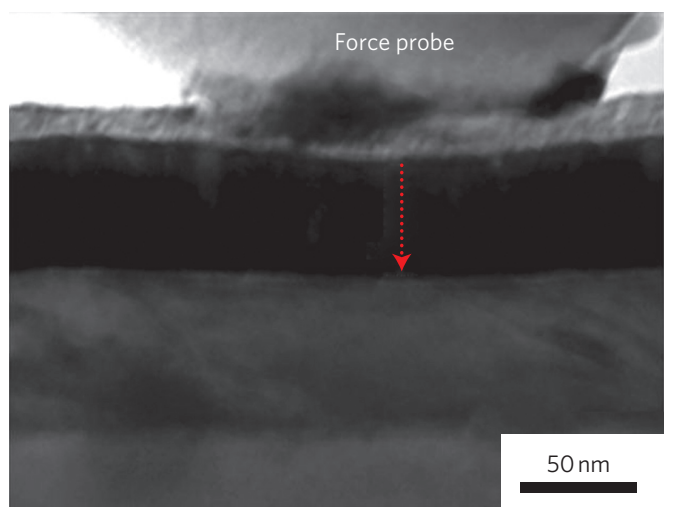

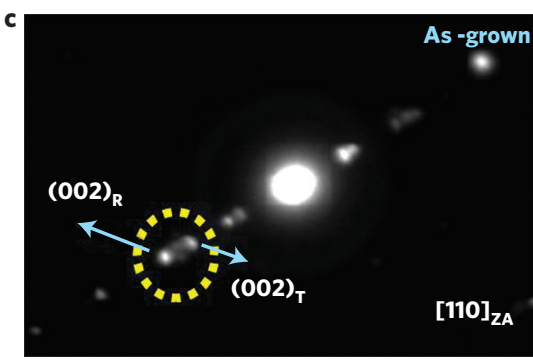
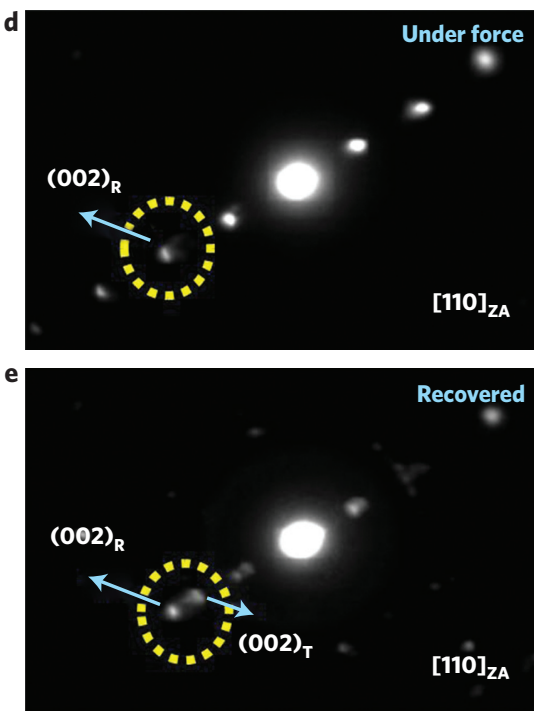

Figure 4 | In situ mechanical TEM. a,b, Bright-field TEM images before (a) and after (b) application of a mechanical force by a nanoindenter. c-e, Evolution of SAD patterns before application of a mechanical force (c), with eventual increase of the mechanical force to $30 \mu \mathrm{N}$ (d), and after release of this mechanical force (e). The change in image contrast and the SAD patterns clearly show the reversible phase transition between mixed and pure R-phase through the application of a mechanical field. 


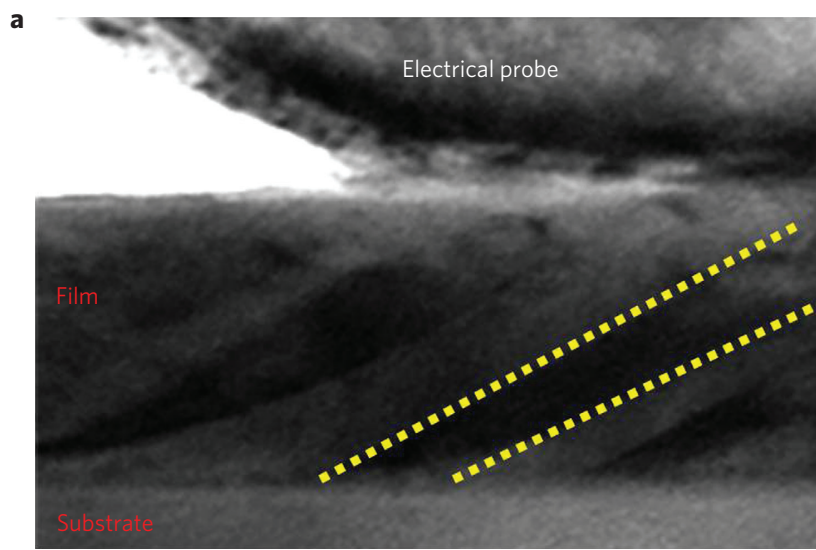

b
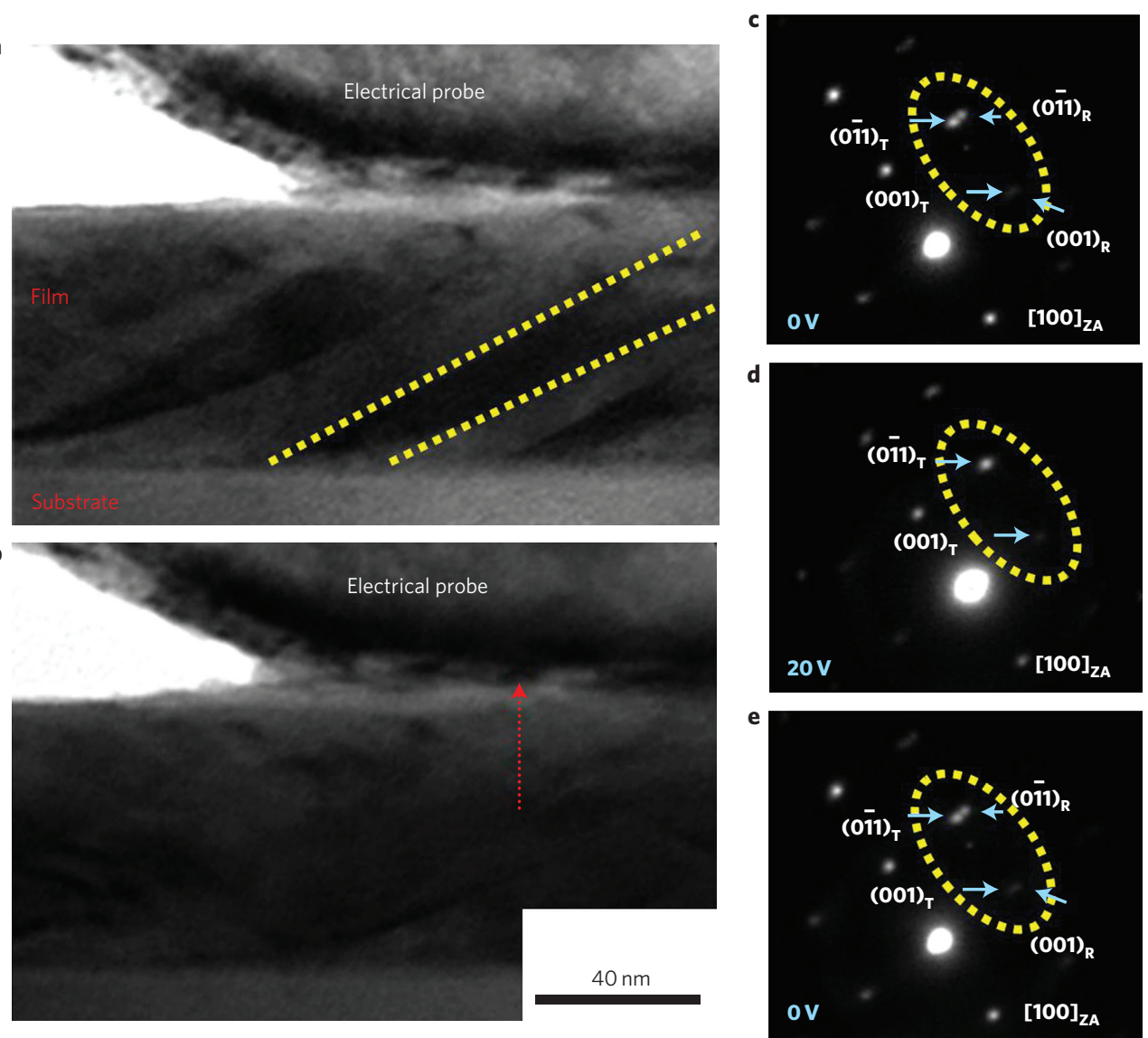

Figure $\mathbf{5}$ | In situ electrical TEM. a,b, Bright-field TEM images before (a) and after (b) application of a electric field by the nanoindenter. c-e, Evolution of SAD patterns under probe voltages of $0 \mathrm{~V}(\mathbf{c}), 20 \mathrm{~V}(\mathbf{d})$ and back to $\mathrm{OV}(\mathbf{e})$. Bright-field images and evolution of the diffraction patterns indicate the reversible phase transition between mixed and pure T-phase under a probe electric field.

$\sim 100 \mathrm{~nm}$ (defined as a strain of $\sim 5 \%$ ), under application of a d.c. bias of $+15 \mathrm{~V}$. The mixed phase is recovered by application of a reversed d.c. bias of $-8 \mathrm{~V}$. The surface deformation in these three states is shown in Fig. 3d. It is noteworthy that this is a completely reversible process, as illustrated in Fig. 3e, which shows the surface displacement as a function of alternating positive and negative d.c. bias applied to the same location.

To understand the details of how these changes occur, we carried out transmission electron microscopy (TEM) studies, coupled with local electrical and mechanical stimuli (for details, see Methods). Stripe-like features (Fig. 4a) are indicative of the mixed-phase structure and vanish completely upon application of a normal mechanical force of up to $\sim 30 \mu \mathrm{N}$ using a nanoindenter (Fig. $4 \mathrm{~b}$ ), indicating a pressure-induced phase transition from the mixed phase to pure R-phase. The reversibility of the phase transition can be demonstrated by the recovery of the mixed-phase structure once the mechanical force is removed. Proof for this reversible phase evolution can be derived from selected area diffraction (SAD) patterns (Fig. 4c-e) obtained before, during and after application of the mechanical force. The mixed-phase structure is identified by a clear splitting of the diffraction spots in Fig. 4c. When this region completely converts to a single phase, the SAD pattern shows only one spot (Fig. 4d), which can be identified as arising from the $\mathrm{R}$-phase. When the nanoindenter is retracted, the mixed phase returns, as illustrated by the split diffraction spots in Fig. 4e.

It is also possible to drive the phase transition with an electric field. To electrically probe the BFO film directly at the nanoscale, the platinum top electrode (used to reduce the effects of damage during the milling process) was removed by the nanoindenter. The reversible motion of the phase boundary can be observed in real time during the application of a voltage of up to $\pm 20 \mathrm{~V}$ (Fig. 5a,b). In the asgrown state, the mixed phase results in a splitting of the $0 \overline{1} 1$ diffraction spots (Fig. 5 c). On application of $+20 \mathrm{~V}$, the split spots are replaced by a single spot (Fig. $5 \mathrm{~d}$ ). It is particularly noteworthy that this $0 \overline{1} 1$ diffraction spot is consistent with the T-phase, not the R-phase. Upon returning to $0 \mathrm{~V}$, the split spots re-emerge (Fig. 5e). See Supplementary Figs S2 and S3 for the quantitative reversible surface displacements resulting from the mechanically $(\sim 3.6 \mathrm{~nm})$ or electrically $(\sim 3.2 \mathrm{~nm})$ induced phase transitions in a mixed-phase film with a thickness of $\sim 70 \mathrm{~nm}$ (these changes are also schematically described in Supplementary Fig. S4). The dynamic details of the phase evolution as a function of mechanical or electric field are also presented in two movie clips in Supplementary Document S6.

The local AFM and TEM studies clearly illustrate the ability to completely convert the mixed-phase films to pure R- or T-phases with either mechanical or electrical stimuli. Note that these promising results and our understanding of the details of this phase evolution are still at an early stage. This work parallels the development of an understanding of the true structural nature of MPBs in classical systems, which, to this day, remains a matter of much discussion. Regardless, it is clear, as in compositionally driven MPBs, that a number of factors including strain, thickness, magnitude of the electric field and/or stress are involved in determining the effects observed in these strain-driven boundaries. Supplementary Fig. S5 schematically captures parts of the field-induced phase evolution, corresponding to the experimental results reported here. The free energy of the system and the corresponding $\mathrm{c} / \mathrm{a}$ (the ratio of out-of-plane and in-plane lattice parameters) are modulated by external stress $\sigma$ and 
electric field $E$. We have observed that compressive stress normal to the film surface leads to stabilization of the R-phase, and that simply removing the compressive stress leads to the recovery of the mixed phase. In the current experimental setup, unfortunately, we cannot directly impose a corresponding tensile stress, but these results suggest that if this were possible one would be able to directly convert the mixed phase into a pure T-phase. We can therefore envisage a combined electric field/mechanical stress cycle that would allow one to directly convert between the R- and T-phases and obtain correspondingly large mechanical responses. Although the transition from one phase to the other seems to be difficult in large areas due to the clamping effect of the substrate, thus yielding an apparent $d_{33}$ value that is not as high as in other composition-controlled MPBs in bulk-such as $\mathrm{Pb}\left(\mathrm{Zr}_{x} \mathrm{Ti}_{1-x}\right) \mathrm{O}_{3}, \mathrm{Ba}\left(\mathrm{Zr}_{0.2} \mathrm{Ti}_{0.8}\right) \mathrm{O}_{3}-\left(\mathrm{Ba}_{0.7} \mathrm{Ca}_{0.3}\right) \mathrm{TiO}_{3}$, and so on-the observed large strain value without dielectric breakdown and the understanding of the origin of this nanoscale phase transition are valuable for designing new and improved materials for next-generation devices. For example, higher $d_{33}$ values could be obtained by reducing the lateral dimensions of the capacitors to remove the clamping effect ${ }^{22}$ and/or reducing its coercive field through chemical doping ${ }^{29}$.

In summary, a reversible, large electric-field-induced strain of over $5 \%$ is achieved in a BFO film consisting of a nanoscale mixture of T- and R-like phases. Localized nanoscale switching dynamics analysis further suggests a large piezoresponse as a consequence of the phase interconversion in an applied electric field. In situ electromechanical TEM measurements directly unveil the origin of this large strain. The reversible, large electromechanical coupling demonstrates the potential of this nanoscale phase boundary as a substitute for lead-based materials in future micro/nanoactuators.

\section{Methods}

Epitaxial $\mathrm{BiFeO}_{3}$ (BFO) films with a mixture of R- and T-phases $(\sim 120-150 \mathrm{~nm})$ and pure T-phase $(\sim 20 \mathrm{~nm})$ were grown on $(001) \mathrm{LaAlO}_{3}$ substrates with ( $\mathrm{La}, \mathrm{Sr}$ ) $\mathrm{CoO}_{3}$ bottom electrodes $(\sim 5-10 \mathrm{~nm}$ ) using pulsed laser deposition and chemical vapour deposition. Growth details can be found in References 3 and 30.

For quantitative electromechanical measurements, a platinum top electrode with a thickness of $\sim 40 \mathrm{~nm}$ and a diameter of $32 \mu \mathrm{m}$ was deposited by sputtering to form a capacitor structure. Piezoelectric properties were characterized by an AFM-based setup (Veeco-DI, equipped with a Nanoscope IV controller) under ambient conditions using a commercially TiPt-coated silicon tip (MikroMasch), which had the same potential as the platinum top electrode during measurements. A driving voltage of $1 \mathrm{~V}_{\mathrm{pp}}$ at $6.39 \mathrm{kHz}$ was applied to the tip. For surface displacement measurements, the probe poling rate was kept at $0.5 \mu \mathrm{m} \mathrm{s}^{-1}$. The AFM images were acquired in contact mode with a scanning rate of $1 \mu \mathrm{m} \mathrm{s}^{-1}$.

The samples for in situ electro-mechanical TEM experiments were prepared by FIB to mill out two opposing trenches on the film. Following this process, a thin window was left on the substrate that was electron-transparent (less than $\sim 100 \mathrm{~nm}$ ). An important issue for FIB sample preparation was to protect the sample from damage caused by the incident $30 \mathrm{kV} \mathrm{Ga}^{+}$ion beam. In our process, a $20,000 \mathrm{pA}$ ion beam current was used for large-area milling at the beginning of the trenches, with increasingly smaller currents used closer to the window. By using a $10 \mathrm{pA}$ ion beam current during the final milling stage, the amount of damage to the sample was kept as small as could effectively be achieved. In situ mechanical and electrical tests were performed with a Hysitron nanoscale electrical contact resistance (nanoECR) picoindenter system in a JEOL $3010(300 \mathrm{kV})$ TEM, equipped with a boron-doped diamond tip $(\sim 150 \mathrm{~nm})$. A biasing sample stage in the nanoECR system was used to apply a bias voltage to the sample using a Keithley 2602A source-meter, which drove the electric flow through the tip and sample.

\section{Received 13 September 2010; accepted 30 November 2010;} published online 16 January 2011

\section{References}

1. Park, S. E. \& Shrout, T. R. Ultrahigh strain and piezoelectric behavior in relaxor based ferroelectric single crystals. J. Appl. Phys. 82, 1804-1811 (1997)

2. Liu, W. et al. Large piezoelectric effect in Pb-free ceramics. Phys. Rev. Lett. 103, 257602 (2009).

3. Zeches, R. J. et al. A strain-driven morphotropic phase boundary in $\mathrm{BiFeO}_{3}$. Science 326, 977-980 (2009).

4. Hathaway, K. \& Clark, A. E. Magnetostrictive materials. MRS Bull. 18, 34-41 (1993)
5. Ren, X. \& Otsuka, K, Origin of rubber-like behavior in metal alloys. Nature 387 579-582 (1997).

6. Chernenko, V. A. et al. The development of new ferromagnetic shape memory alloys in Ni-Mn-Ga system. Scripta metallurgica et materialia 33, 1239-1244 (1995).

7. Xu, Y. H. Ferroelectric Materials and Their Applications 109 (North-Holland, 1991).

8. Dkhil, B. et al. Local and long range polar order in the relaxor-ferroelectric compounds $\mathrm{PbMg}_{1 / 3} \mathrm{Nb}_{2 / 3} \mathrm{O}_{3}$ and $\mathrm{PbMg}_{0.3} \mathrm{Nb}_{0.6} \mathrm{Ti}_{0.1} \mathrm{O}_{3}$. Phys. Rev. B 65, 024104 (2002).

9. Noheda, B. et al. Polarization rotation via a monoclinic phase in the piezoelectric $92 \% \mathrm{PbZn}_{1 / 3} \mathrm{Nb}_{2 / 3} \mathrm{O}_{3}-8 \% \mathrm{PbTiO}_{3}$. Phys. Rev. Lett. 86, 3891-3894 (2001).

10. Noheda, B. et al. A monoclinic ferroelectric phase in the $\mathrm{Pb}\left(\mathrm{Zr}_{1-x} \mathrm{Ti}_{x}\right) \mathrm{O}_{3}$ solid solution. Appl. Phys. Lett. 74, 2059-2061 (1999).

11. Ahart, M. et al. Origin of morphotropic phase boundaries in ferroelectrics. Nature 451, 545-548 (2008).

12. Wu, Z. \& Cohen, R. E. Pressure-induced anomalous phase transitions and colossal enhancement of piezoelectricity in $\mathrm{PbTiO}_{3}$. Phys. Rev. Lett. 95, 037601 (2005).

13. Saito, Y. et al. Lead-free piezoceramics. Nature 432, 84-87 (2004).

14. Ederer, C. \& Spaldin, N. A. Effect of epitaxial strain on the spontaneous polarization of thin film ferroelectrics. Phys. Rev. Lett. 95, 257601 (2005)

15. Béa, H. et al. Evidence for room-temperature multiferroicity in a compound with a giant axial ratio. Phys. Rev. Lett. 102, 217603 (2009).

16. Chmielus, M. et al. Giant magnetic-field-induced strains in polycrystalline Ni-Mn-Ga. Nat. Mater. 8, 863-866 (2009).

17. Sozinov, A. et al. Giant magnetic-field-induced strain in NiMnGa seven-layered martensitic phase. Appl. Phys. Lett. 80, 1746-1748 (2002).

18. Zavaliche, F. et al. Multiferroic $\mathrm{BiFeO}_{3}$ films: domain structure and polarization dynamics. Phase Transitions 79, 991-1017 (2006).

19. Fujino, S. et al. Combinatorial discovery of a lead-free morphotropic phase boundary in a thin-film piezoelectric perovskite. Appl. Phys. Lett. 92, 202904 (2008)

20. Du, X. et al. Crystal orientation dependence of piezoelectric properties of lead zirconate titanate near the morphotropic phase boundary. Appl. Phys. Lett. 72, 2421-2423 (1998)

21. Chen, H. D. et al. Electrical properties' maxima in thin films of the lead zirconate-lead titanate solid solution system. Appl. Phys. Lett. 67, 3411-3413 (1995).

22. Nagarajan, V. et al. Dynamics of ferroelastic domains in ferroelectric thin films. Nat. Mater. 2, 43-47 (2002).

23. Hong, S. et al. Nanoscale piezoresponse studies of ferroelectric domains in epitaxial $\mathrm{BiFeO}_{3}$ nanostructures. J. Appl. Phys. 105, 061619 (2009).

24. Nath, R. et al. Effects of cantilever buckling on vector piezoresponse force microscopy imaging of ferroelectric domains in $\mathrm{BiFeO}_{3}$ nanostructures. Appl. Phys. Lett. 96, 163101 (2010)

25. Durkan, C. et al. Probing domains at the nanometer scale in piezoelectric thin films. Phys. Rev. B 60, 16198-16204 (1999).

26. Foster, C. M. Single-crystal $\mathrm{Pb}\left(\mathrm{Zr}_{x} \mathrm{Ti}_{1-x}\right) \mathrm{O}_{3}$ thin films prepared by metal-organic chemical vapor deposition: systematic compositional variation of electronic and optical properties. J. Appl. Phys. 81, 2349-2357 (1997).

27. Palkar, V. R. et al. Observation of saturated polarization and dielectric anomaly in magnetoelectric $\mathrm{BiFeO}_{3}$ thin films. Appl. Phys. Lett. 80, 1628-1630 (2002).

28. Xu, G. et al. Phase instability induced by polar nanoregions in a relaxor ferroelectric system. Nat. Mater. 7, 562-566 (2008).

29. Chu, Y. H. et al. Low voltage performance of epitaxial $\mathrm{BiFeO}_{3}$ films on $\mathrm{Si}$ substrates through lanthanum substitution. Appl. Phys. Lett. 92, 102909 (2008).

30. Yang, S. Y. et al. Metalorganic chemical vapor deposition of lead-free ferroelectric $\mathrm{BiFeO}_{3}$ films for memory applications. Appl. Phys. Lett. 87, 102903 (2005).

\section{Acknowledgements}

The work at Berkeley was supported by the Director, Office of Science, Office of Basic Energy Sciences, Materials Sciences Division of the US Department of Energy (contract DE-AC02-05CH11231). The authors acknowledge support from the National Center for Electron Microscopy, Lawrence Berkeley National Laboratory, which is supported by the US Department of Energy (contract DE-AC02-05CH11231). The work at National Chiao Tung University was supported by the National Science Council (contract 099-2811-M009-003). J.S. acknowledges support from the Alexander von Humboldt Foundation.

\section{Author contributions}

J.X.Z., R.R., B.X., and A.M.M. conceived and designed the experiments. J.X.Z. and B.X performed the experiments. J.X.Z., B.X., Q.H., J.S., P.Y., Y.H.C., L.W.M. and A.M.M. analysed the data and helped revise the manuscript. R.J.Z., P.Y., S.Y.Y. and C.H.W. contributed materials. J.X.Z., J.S. and R.R. co-wrote the paper.

\section{Additional information}

The authors declare no competing financial interests. Supplementary information accompanies this paper at www.nature.com/naturenanotechnology. Reprints and permission information is available online at http://npg.nature.com/reprintsandpermissions/. Correspondence and requests for materials should be addressed to J.X.Z. 